\title{
Verantwortung haben Ärzte reichlich. Und Führung?*
}

Ludwig Hasler

* Der Beitrag basiert auf einem Referat, das der Autor am 25. Oktober 2012 an der Plenarversammlung des Schweizerischen Instituts für ärztliche Weiter- und Fortbildung SIWF in Bern gehalten hat.
Vor Jahresfrist kritisierte ich die Überschätzung der Weiterbildung - genauer: die Gefahr der Entmündigung durch permanente Bildung. Denn fachliche Bildung betreibt den Glauben an Erfahrungen anderer - und dieser anschwellende Glaube an Fremderfahrung überflutet leicht die eigene Erfahrung der Ärztin, so dass (Achtung Satire!) am Ende die weitergebildete Ärztin funktioniert wie eine mit Studienkenntnissen vollgepackte Speicherplatte, wie ein Medizin-Roboter, der den jüngsten Stand des vermeintlich sakrosankten Wissens auf akute Fälle appliziert, was therapeutisch unergiebig wäre (weil kein Patient ist wie der andere) und wissenschaftlich naiv (weil jedes Wissen vorläufig bleibt). sehen wenig Chance zu tun, was sie eigentlich tun sollten (Erfahrung weitergeben); Weiterbildung schneidet in ihrem Urteil schlecht ab, sie sei ein Ritual von Pharma und Wissenschaft, ein «Selbstdarstellungs-Zirkus» für Wissenschaftler - statt für behandelnde Ärzte, Evidence based statt Eminence based, der praktische Nutzen sei gering, gemeinschaftsbildend einzig der Kampf gegen den Schlaf ... Die Kritik der Ärzte war drastisch. Repräsentativ? Keine Ahnung. Sie stammt von Ärzten, die über den Tellerrand hinaussehen.

Zweite Beobachtung. Nobelpreis für Medizin. Geht dieses Jahr an zwei Stammzellforscher. Ausnahmsweise sogar für mich einleuchtend: Der eine (Brite)

\section{«Wollen Sie nicht als Vollstrecker polymorpher Bedürfnisse enden, müs- sen Sie sich einen Kanon ärztlichen Selbstverständnisses verschreiben.»}

Das war letztes Jahr. Die Kritik blieb wirkungslos, weshalb ich heute für Weiterbildung plädiere - nicht für medizinische Spezialitäten (die läuft von selbst), auch nicht für «nicht fachspezifische Lernziele» (läuft unter dem Boom-Label ebenfalls ohne mich, als flankierende Trainingseinheit in Sekundärtugenden, «persönlichkeitsstützende» Exerzitien, soft skills usw.). Nein, ich plädiere für Fortbildung in ärztlichem Selbstverständnis: als Nachhilfe für geklärtes Selbstbewusstsein, gestärkte Branchen-Identität. In turbulenten Zeiten muss eine Branche sich klarmachen, was sie will. Schon gar eine Branche wie die ärztliche, die von allen Seiten beansprucht, gern instrumentalisiert, auch ideologisiert wird. Wollen Sie nicht als willfährige Vollstrecker polymorpher Bedürfnisse enden, müssen Sie sich selber einen Kanon ärztlichen Selbstverständnisses verschreiben.

Wie ich zu dieser Behauptung komme? Ich bin hier der Laie. Doch allein der Monat Oktober lieferte mir drei Beweggründe.

Zunächst dozierte ich (im Rahmen eines universitären MBA) Philosophie für (meist jüngere) Kaderärzte. Was sagen die arrivierten Ärzte (die ihren Samstag der Bildung widmen!) über ihre Stellung im Beruf? Zwiespältiges. Sie fühlen sich unsouverän im eigenen ärztlichen Handeln (dominiert von Administration, von Regularien wie DRG usw.); Chefärzte fand einen Jungbrunnen für Zellen, der andere (Japaner) machte ihn zur Basis neuer Therapien. Toll. Da liegt Zukunft in der Luft. «Alleskönner»-Zellen, genial. Ist das Medizin? Zellbiologie. Froschexperimente. Für Medizin ausgezeichnet wird ein Wissen, dass nicht aus der Medizin kommt, schon gar nicht vom Krankenbett. Ergo: Wird Medizin wissenschaftlich zur Bedürfnisbranche, die sich ihr Wissen nicht mehr selber besorgt?

Dritte Beobachtung. In Deutschland demonstrieren/streiken Ärzte. Parole: «Holen wir unsere Selbstbestimmung zurück!» War sie ausgewandert? Wohin? In Ökonomie? Politik? Zu den Krankenkassen? Läuft in der Schweiz alles harmloser? Positiver? Aargauer Zeitung: «Das Kantonsspital Aarau hat seine Geschäftsleitung auf zwölf Personen aufgestockt, damit auch die Ärzte mitreden können.» Also doch. Ärzte reden mit - im Machtzentrum. Genau, was ich (siehe Titel) wünsche: Ärzte, die sich nicht nur in der Verantwortung sehen, sondern auch in der Führung?

Drei Oktober-Beobachtungen, Tonart skeptisch. Der Arzt wird abhängiger: zum Anwender eines (biologisch-technischen) Wissens, das nicht aus ärztlicher Tätigkeit stammt, nach Regeln, die extern gebildet werden (Ökonomie, Politik, Industrie). Jetzt eine Spur systematischer. Erst mache ich mich unbeliebt 


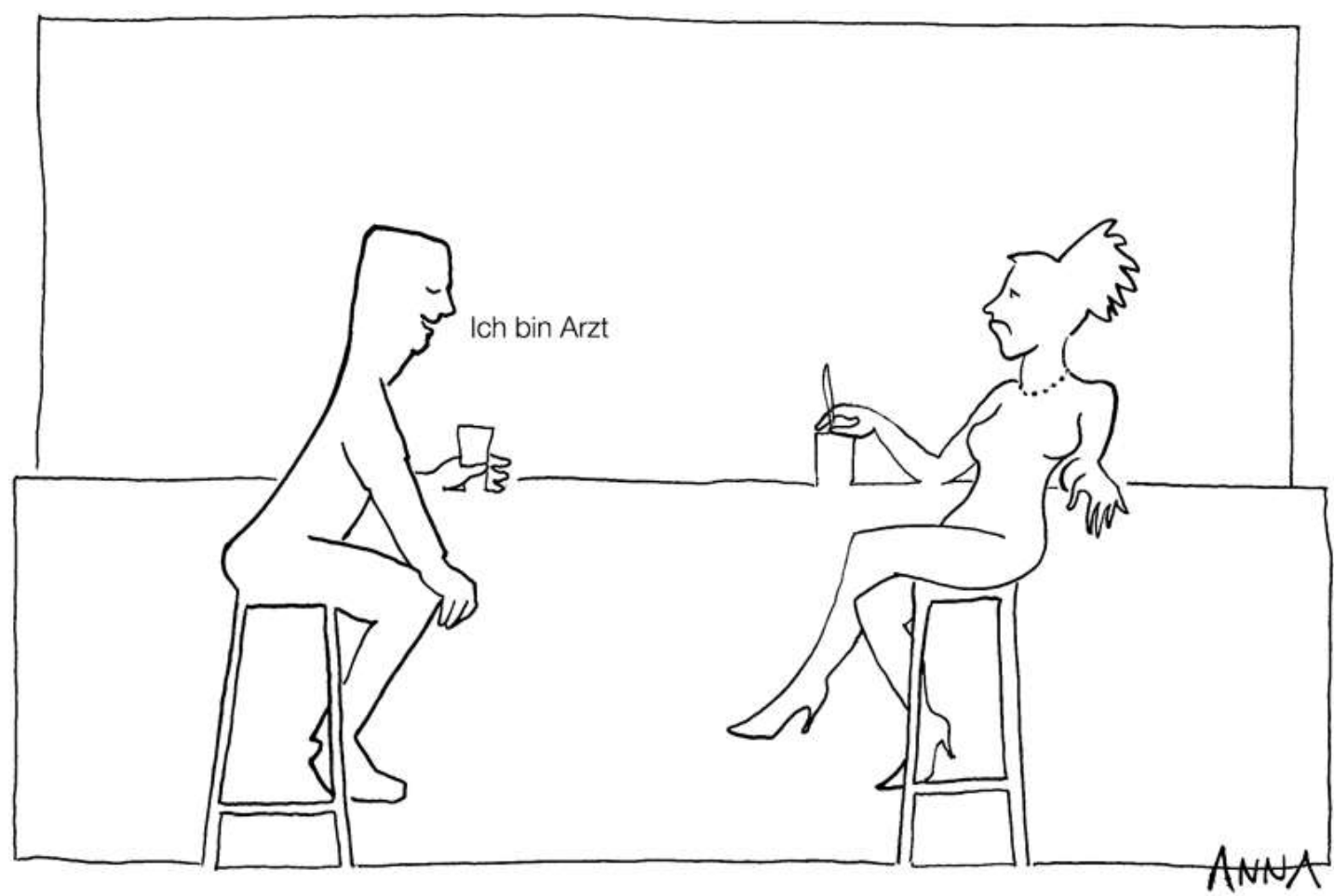

Auch eine Folge der Erosion von Ansehen und Macht der Ärzte ...

in Runde 1: Zur Erosion ärztlicher Souveränität oder: Vom Hausherrn zum Lakaien? Danach denke ich darüber nach, wie der Trend zu bremsen/stoppen/kehren wäre, Runde 2: Zur Revitalisierung der Souveränität - oder: Von der Eigenart ärztlichen Handelns.

\section{Erosion ärztlicher Souveränität - oder: vom Hausherrn zum gehobenen Lakaien?}

Einst war der Arzt Herr seiner Wirkungskreise - in Spital, Praxis, Universität. Ein freier Unternehmer im Dienste des Menschenwohls. Heute erwacht er aus dem Traum des Unternehmers/Freiberuflers. Freiberufler entscheiden selber: über geeignete Methoden, über das Ausmass der Anwendung, über Vergütung. In all diesen Fällen wächst der Einfluss externer Steuerungsinstanzen: Biologie/Pharma, Ökonomie, Politik. Konsequenz: Selbständigkeitsverluste - in deren Folge Einbussen an Kompetenz, an Einkünften, an Prestige. Die Entwicklung steckt vermutlich noch in der harmlosen Phase. Wie wichtig es gerade in dieser Phase ist, den Verlauf genau zu beobachten, das wissen Ärzte berufshalber. Also.

Gesellschaftlich verliert der Arzt seine traditionelle Sonderstellung. Es passiert, was andere Branchen früher erlebten: die Normalisierung der einst exklusiven medizinischen Profession. Der Arzt wird Mediziner, und Mediziner ist ein Beruf wie andere. Also Schluss mit der Ärzteschaft als hermetischem Club, als selbstreferentieller Gruppe, die sich traditionell mit sich selbst verglich. Obwohl der Beruf noch immer prägt wie kaum ein anderer: die unausweichli- che Überforderung an der Grenze zwischen Leben und Tod, existentielle Ausnahme als beruflicher Normalfall. Beginnt schon mit dem Studium: Kaum ein anderes Fach hat solch harte Prüfungen, so viel Stoff, so viel Druck, danach die Assistenzzeit, endlose Schichten, strenge Hierarchie, fachlich dauernd am Limit. Dazu die Erwartungen der Gesellschaft. Wer Arzt ist, muss auch Held sein, siehe TV-Serien, siehe «Lost» (zwei Dutzend Menschen überleben Flugzeugabsturz, retten sich auf Insel, wer ist Anführer? Der Arzt). Die Klischees werden verlängert - und kontrastiert durch Abwertungen des Heldenstatus (Bürokratie, Kosten-Kontrolle, Laienaufstand dank Internet usw.). - Nichts Dramatisches. Aber doch Ansätze einer Entwicklung, die irgendwann auf die Frage hinausläuft: Wer tut sich etwas an? Leute mit Helfersyndrom? Mit masochistischen Neigungen? Mit unerschütterlichem Idealismus?

Medizinisch wird der Arzt zum Fremdling in der Medizin. Sagt Paul U. Unschuld, der Münchner Medizinhistoriker, in seinem gleichnamigen Buch. Seine Argumentation: Medizin werde organisiert nach Kriterien ökonomischer Planungs- und Kontrollvernunft. Nicht ärztliche Erfahrung im Umgang mit Leidenden bewirkte zum Beispiel die Fallpauschale; es war ökonomische Routine, die Usanzen der Autoreparaturwerkstätte in Krankenhäuser überträgt. Noch das Wissen, mit dem Ärzte professionell handeln, kommt kaum noch von Ärzten. Der Nobelpreis ist nur der schlagendste Beleg. Wer erhielt ihn in den letzten 20 Jahren? Biochemiker, Biologen, Pharmakologen. Wird medizinisches Wissen Fremd- 
wissen? Biologisches, zellbiologisches, molekularbiologisches, biochemisches Wissen? Unter den letzten zwanzig Preisträgern hatten immerhin noch sechs Medizin studiert, zwei waren in einer medizinischen Fakultät beschäftigt. Wie viele standen am Krankenbett? Keiner.

\section{Das Wissen, mit dem Ärzte professionell handeln, kommt kaum noch von Ärzten.}

Auch das ärztliche Handeln büsst Souveränität ein. Zwar gerät - in der sog. Wissensgesellschaft - jeder Beruf in Abhängigkeit von Wissenschaft und Forschung. Doch andere Branchen nutzen einschlägige wissenschaftliche Studien - oder eben nicht. In der Wirtschaft etwa fällt keinem Manager in heikler Lage ein, im ökonomischen Lehrbuch nachzuschlagen. Umgekehrt vertraut kein Verwaltungsrat Ökonomiewissenschaftlern die Führung einer Firma an; man hält sie für Eierköpfe, die nach Gesetzmässigkeiten forschen, die Praxis aber folgt, da ist man sich einig, anderen Entscheiden, nämlich situativen. Auch in der pädagogischen Provinz gilt: Grau ist alle Theorie - die gute Lehrerin erfindet ihren Unterricht täglich selbst. Dass mein Arzt sich nicht gar so souverän aufführt, ist mir zwar recht. Aber muss darum ärztliches Handeln die Trennlinie zwischen Theorie und Praxis gleich verwischen, alle Erfahrungsgeleitetheit der Praxis sozusagen auf dem Altar der Wissenschaft opfern?

\section{«45 Fachgesellschaften - da ist ja die Erosion des Arztes programmiert.»}

Ist diese Entwicklung - Erosion der Souveränität, vom Hausherrn zum Lakaien - schlimm? Oder normal? Der Preis der Professionalisierung? 45 Fachgesellschaften - da ist ja die Erosion des Arztes programmiert, verläuft sich in Dutzende von Spezialitäten, die (zum Teil) mit der traditionellen ärztlichen Rolle kaum etwas zu tun haben (weil sie eher hochspezialisierte Handwerker/Künstler sind, oder weil sie sich gar nicht als Heilberufler verstehen, da sie nicht länger Krankheiten therapieren, sondern Fakten optimieren). Überdies ist der Status Hausherr vielen $\mathrm{zu}$ anstrengend, sie wollen sich nicht am Beruf aufhängen; der Lakai hat seinen Job, sein Salär, seinen Tagesbefehl, er erledigt ihn, basta, danach ist er frei. So läuft die subjektive Seite der Professionalisie-
rung/Differenzierung. Gibt es bald einen Bachelor Kniegelenk, einen Master Darmspiegelung? Wäre es das Ende «ärztlicher Souveränität»«? Definitiv nur «Dienstleistungserbringer»? Hochkompetent - und subaltern?

Ist Souveränität anachronistisch? Vergangenheitsbeschönigung plus Gegenwartsverkennung? Mal sehen:

\section{Revitalisierung der Souveränität - oder: von der Eigenart ärztlichen Handelns}

Wir können es drehen, wie wir wollen: Entweder wir codieren unsere Standards selber - oder andere tun es für uns. Entweder bestimmen Ärzte selber die Codes, unter denen sie handeln, oder diese Codes werden von aussen verordnet. Darum finde ich, sollte ärztliche Fortbildung - immer auch - etwas tun für die Autonomie der Branche. Sie bewegte sich damit in einer langen Tradition.

Ärzte legten zu allen Zeiten Wert auf die Selbstverpflichtung ihres Standes. Der hippokratische Eid wurde ihnen nicht von aussen auferlegt. Sie kennen die Worte: «Ich werde ärztliche Verordnungen treffen zum Nutzen der Kranken nach meiner Fähigkeit und meinem Urteil, hüten aber werde ich mich davor, sie zum Schaden und in unrechter Weise anzuwenden ...» So steht es in jenem berühmten Eid aus der Antike. Heute, 2500 Jahre später, schwören Ärzte nicht mehr bei den Göttern Apollon und Asklepios. Doch die wichtigsten Gedanken dieser Ethik gelten noch immer: das Primat des Patientenwohls. Die Schweigepflicht. Das Euthanasieverbot. Das primum non nocere: das Gebot, einen Eingriff eher zu unterlassen, als künftige Beeinträchtigungen, gar den Tod zu riskieren. Nach dem Zweiten Weltkrieg wurde der hippokratische Eid vom Weltärztebund zeitgemäss formuliert. Dieses «Genfer Gelöbnis» betont ausdrücklich, dass Ärzte ihre Patienten unabhängig von sozialer Stellung, Geschlecht oder anderen Zugehörigkeiten behandeln sollen - und ihre medizinischen Kenntnisse selbst dann anwenden sollen, wenn sie bedroht sind von Kräften, die «im Widerspruch zu den Geboten der Menschlichkeit» operieren. Wer sind heute die Kräfte wider die Menschlichkeit? Taliban? Der Vatikan? DRG-Regime? Tabakindustrie? Tugendterror - Rauchverbot im Altersheim?

Jedenfalls scheint Arzt doch nicht ein Beruf wie andere zu sein. Er hat eine Besonderheit: die Wahrung eines unbedingten Gutes - der Schutz des Kranken. Andere Berufsgruppen (Schreiner, Wirte, Transport) bewirtschaften Bedürfnisse ihrer Klientel. Ärzte tun das auch. Aber sie müssen - anders als andere Branchen die Bedürfnisse der Kranken nicht nur bedienen, sie müssen sie auch schützen gegen den Zugriff anderer Mächte. Dieser Schutz lässt sich nicht pragmatisch regeln, er muss absolut gelten, auch wenn manche pragmatischen Aspekte gegen ihn sprechen. Darum darf ärztliches Handeln sich nicht (nur) pragmatisch nach anderen Steuerungsinstanzen richten, es muss sein 
Prinzip in sich finden. - Darüber liesse sich in mindestens drei Schleifen nachdenken:

Erstens müssen Ärzte ihre Rolle im Markt bestimmen. Sind sie Marktteilnehmer wie andere Anbieter - oder vertreten sie (auch) ein Gut, selbst wenn der Markt es ignoriert? Nicht nur steckt Arztsein wie kaum ein zweiter Beruf stets im Zwiespalt, die Sorge um die Not der anderen mit der Sorge um die eigenen Bedürfnisse zu verbinden. Wichtiger ist: Ärzte sind schon in friedlichen Zeiten Médecins sans frontières. Sie verteidigen die Würde der Kranken gegen alle Widersacher - durch die Unkäuflichkeit ihrer Dia-gnose, durch die Unkorrumpierbarkeit ihrer Therapie. Was Krankheit ist (und was nicht), das wollen viele beeinflussen (Parteien, Industrien ...). Entscheidend ist, dass Krankheit (und Therapie) am Leid der betroffenen Menschen orientiert bleibt und nicht an politischen oder wirtschaftlichen Inter-essen. Wer soll das leisten, wenn nicht der Arzt? Keiner weiss wie er, was Kranke leiden, was sie wollen, brauchen. Der Arzt, der einzig legitime Anwalt des kranken Menschen.

Zweitens sollten Ärzte eine genuin ärztliche Heilkunde verteidigen. Nichts gegen das aufdatierte Studienwissen. Aber wo bleibt da das Menschenbild? Welchen Menschenschlag haben Ärzte vor Augen? Was vertreten sie? Das Leben? Aber was für eines? Sie zogen mit in den Kreuzzug gegen das Rauchen. Okay. Was bedeutet das für die Philosophie vom Leben? Leben gleich Schonen? Bewahre uns vor allem Übel - oder: Sorget euch nicht? Denken Ärzte über solche Dinge noch nach? Wäre zwingend. Was macht das «gute Leben» aus? Fitness oder Verschwendung? Bravheit oder Sünde? Vielleicht hat, wer mehr sündigte, ein besseres Alter - vor allem etwas, woran er sterben kann? Während die radikal Properen sich fürs ewige Leben präparieren. Wie reagiert da «der Arzt»? Gar nicht? Jeder soll nach seiner eigenen Façon selig werden? Die Leute suchen aber Rat beim Arzt, nicht nur Pillen. Dazu brauchen Ärzte eine Philosophie menschlichen Lebens. Da Ärzte berufshalber illusionsfreier sind als andere (weil sie zum Beispiel junge Leute sterben sehen, die trotz prima Lebensführung einem Hirntumor zum Opfer fallen), müssten sie Freunde der Endlichkeit sein, für Freundschaft mit der Vergänglichkeit werben, egal, wie antizyklisch das wirkt. Menschen sind antizyklisch.
Eine eigene, genuin ärztliche Heilkunde verteidigen. Philosophisch sekundiert, wie gesagt. Historisch gestützt? Wann wurde Medizin Wissenschaft und gewann Prestige? Als sie sich (in der Antike) vom Handwerk zur Philosophie entwickelte, dann wieder um 1800, als sie zur eigenen Theorie auflief (Schelling!), zur selbständigen Wissenschaft. Bemerkenswert ist die heutige Attraktivität alternativer medizinischer Praktiken, homöopathische, chinesische, indische Medizin. Wie immer man über diese Verfahren denken mag - sie gründen auf Wissen, das von Ärzten für Ärzte geschaffen wurde. Darauf vertrauen die Leute. Noch mehr, wenn diese Methoden ausserhalb der Kassen praktiziert werden, und wenn Diagnose/Therapie keine technischen Ausstattungen verlangen (nur Hühnersuppe!). Das heisst: Es fallen alle Fremdbestimmungen weg (auch darüber, wann und in welchem Ausmasse sie praktiziert werden dürfen und wie viel dafür an Honorar zu entrichten sei). Vielleicht ist das Unbehagen, das nicht wenige Ärzte dazu treibt, sich diesen Verfahren zu öffnen, auch ein Unbehagen an den Strukturen der Fremdbestimmung, die sich um die Anwendung der Schulmedizin herausgebildet haben.

Drittens sollten Ärzte Schlüsselstellen im System Medizin besetzen. Mehr Leadership durch Ärzte. Verantwortung haben sie eh. Dabei lernen sie jede Menge über die durchzogene Conditio humana. Genau diese Erfahrung wäre wichtig für Führungsfiguren im Gesundheitswesen. Aktuell herrscht zu viel Ideologie, Gesundheitspflege wird zur Moral, Leidenschaft zur Charakterschwäche. Ärzte könnten diese Masche durchschauen. Damit wären sie prädestiniert für Führungsjobs - in doppelter Hinsicht: Sie können die akute Gesundheitsideologie pragmatisch diskutieren - und das Leiden der Kranken als undiskutables A \& O des Systems schützen.

Schluss für heute. War kein Programm, nichts als Anregung. Offenbar bin ich nicht allein, wenn ich Fortbildung nicht bloss als Organisation des Wissenstransfers sehe. Der Dichter Rabelais sagte, Bildung dürfe nicht bloss «Fässer füllen» (mit Kenntnissen volltexten), sie müsse «Fackeln anzünden» (Akteure mit dem Feuer der eigenen Souveränität anstecken). Genau das wollte ich sagen. 